\title{
Formation of Distal Bone Elements in Amputated Neonatal Mouse Forelimbs
}

\author{
Hiroyuki Ide* \\ Graduate School of Life Sciences, Tohoku University, Sendai 980-8578, Japan \\ ${ }^{\star}$ Corresponding author: Hiroyuki Ide, Graduate School of Life Sciences, Tohoku University, Sendai 980-8578, Japan; E-mail: ide@m.tohoku.ac.jp
}

Received: February 04, 2019; Accepted: February 13, 2019; Published: February 15, 2019;

\begin{abstract}
Mammalian limbs show little regeneration potency. Mouse and human limbs regenerate only finger tips. However, some Bone Morphogenetic Proteins (BMPs) stimulate the formation of a new radius and ulna on amputated distal elements of neonatal mouse forearms. In this article, we describe the formation of more distal bone elements, carpi and metacarpi-like structures, other than the radius and ulna, after forearm amputation and BMP-7 and hedgehog agonist (Hh-Ag 1.8) treatment. However, since skin and soft connective tissues at the hand level remained in the stumps in these experiments, the small bones may be formed from these hand tissues by BMP and Hh-Ag. Thus, amputation was carried out at the proximal level of the wrist. With BMP and Hh-Ag treatment, the distal ends of the radius and ulna showed bifurcated and segmented bone structures. These results suggest the formation of distal bone elements, carpi and metacarpi, from forearm tissues.
\end{abstract}

Keywords: Mouse limb, regeneration, BMP-7, hedgehog agonist

\section{Introduction}

It is well known that mammalian limbs regenerate only finger tips. In mice, a fingertip is regenerated after amputation and the regeneration is regulated by Bone Morphogenetic Proteins (BMPs) $[1,2]$. We previously reported BMP-induced bone formation in amputated forearms [3]. Furthermore, BMP induced the pattern formation of forearm bones, radius and ulna, in the forelimb of a neonatal mouse from which distal halves of the radius and ulna had been removed previously [4]. A hedgehog agonist (Hh-Ag 1.8) promoted the function of BMP [4]. In an adult mouse, BMP induced bone pattern regeneration of the tibia and fibula in an amputated shank [5]. However, the regeneration stopped at the ankle and no regeneration was observed at the foot level.

In limb regeneration of urodele amphibians, an amputated forearm forms a distal forearm and a hand, new elements. Thus, it would be valuable to try to achieve formation of distal elements in mammalian limbs for regenerative medicine.

In this paper, we describe formation of the carpus and metacarpuslike branched long bone elements on the amputated radius/ulna of forelimbs of neonatal mice and also describe the formation of many skeletal protrusions on the distal ends of the radius/ulna after amputation at the wrist level.

\section{Methods and Materials}

\section{Limb Amputation}

Forelimbs were amputated with a knife at the palm, wrist or distal forearm level. When limbs were amputated at the palm level, proximal halves of metacarpi, all carpi, and distal halves of the radius and ulna were removed with small scissors.

Implantation of a Gelatin Rod Containing BMP-7 and HhAg1.8

A gelatin rod containing $100 \mathrm{ng}$ BMP-7 and $200 \mathrm{ng}$ Hh-Ag1.8 was implanted according to the procedure described by Ide [4]. After the operation, the wound was treated with fibrin glue and surrounded with Tegaderm film.

\section{Alcian Blue/Alizarin Red Staining}

To observe the skeletal pattern, limbs were stained with Alcian blue for cartilage and Alizarin red for bone [6]. Prior to staining, most of the skin was removed and limbs were fixed in $100 \%$ ethanol.

\section{Staining of Sectioned Limbs}

Some limbs were fixed in paraformaldehyde and frozen samples were sectioned. The sections were stained with Alcian blue and/or Elastiica van Giesen [7].

\section{Materials}

Neonatals of DDY mice (SLC, Japan) at 1 to 2 days after birth were used. Procedures for care and use of mice for this study were in compliance with standard operating procedures approved by the Institutional Animal Care and Use Committee of Tohoku University and DARPA (Defence Advanced Research Project Agency).

BMP-7 (Recombinant, Human) was obtained from R\&D Systems, Inc. (Minneapolis, MN). A hedgehog agonist (Hh-Ag1.8) was obtained 
from Curis, Inc. (Lexington MA). Tegaderm Film Roll was obtained from $3 \mathrm{M}$ Healthcare.

\section{Results}

\section{Wrist and palm bone formation on truncated radius and ulna bones}

As reported previously, BMP and $\mathrm{Hh}-\mathrm{Ag}$ promoted formation of the radius-ulna in the forearm of neonatal mice from which distal halves of the radius and ulna had been removed previously [4]. Furthermore, isolated small bones were observed distal to the regenerated radius and ulna (Fig. 1). When only the distal halves of the radius and ulna were removed and the epidermis and soft connective tissues at the wrist and proximal palm levels remained, four to five small long bones were formed on the distal area of the newly formed radius and ulna (Fig. 2). An enlarged view revealed the formation of these small bones on a plane. Cross sections of these newly formed small bones revealed many branched structures on the distal region of forearm bones (Fig 3). This suggests that the formation of wrist and palm bones was induced by the application of BMP-7 and Hh-Ag.
However, the possibility that these bones were formed from the soft connective tissues at the wrist and palm levels remains.

\section{Formation of small distal bones after arm truncation at the wrist level}

The styloid process of the ulna can be observed directly under a dissection microscope on the ulna of a neonatal mouse. Thus, we truncated the arm at the proximal portion of this process. Alizarin red and Alcian blue staining of the truncated arm showed no wrist tissues in the stump (Figure 4A). After 4 days, a gelatin rod containing BMP7 and $\mathrm{Hh}-\mathrm{Ag} 1.4$ was inserted into the space between the amputated bones and wound epidermis. After 10 days, the arms were fixed and stained with Alizarin red and Alcian blue. Four or five protrusions were observed on the top of the truncated radius and ulna (Figure 4B). Some of the protrusions were separated from the radius-ulna bone. No such protrusions were observed on the radius-ulna bone without BMP-7. Only hypertrophy of the truncated portion of the radius-ulna was observed as in the case of amputated forearm bones (Figure $4 \mathrm{C}$ ).
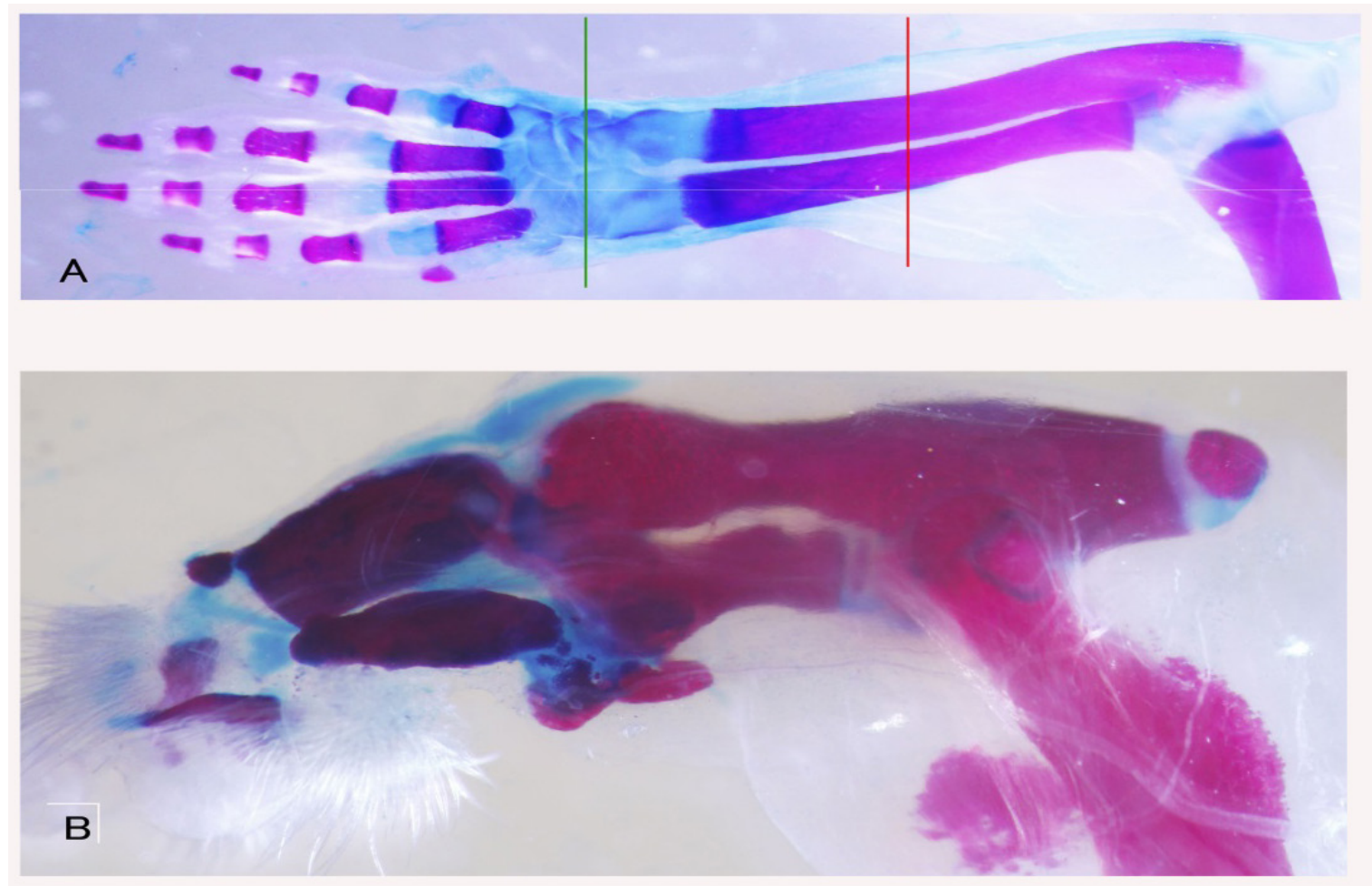

Figure 1. Skeletal pattern formation in a BMP-treated forearm amputated at the palm level. A: Alcian blue and Alizarin red staining of the forearm showing the levels of amputation. The green line shows the amputation level. The distal half of the hand was removed. The red line shows the level of bone amputation. Metacarpi, carpi and distal halves of the radius and ulna were removed with small scissors from the hole of the amputated hand. B: Alcian blue and Alizarin red staining of newly formed bones 14 days after amputation. Two thick bones were formed at the distal side of the stump radius and ulna. Small bones were observed at the tips of these newly formed forearm bones. 


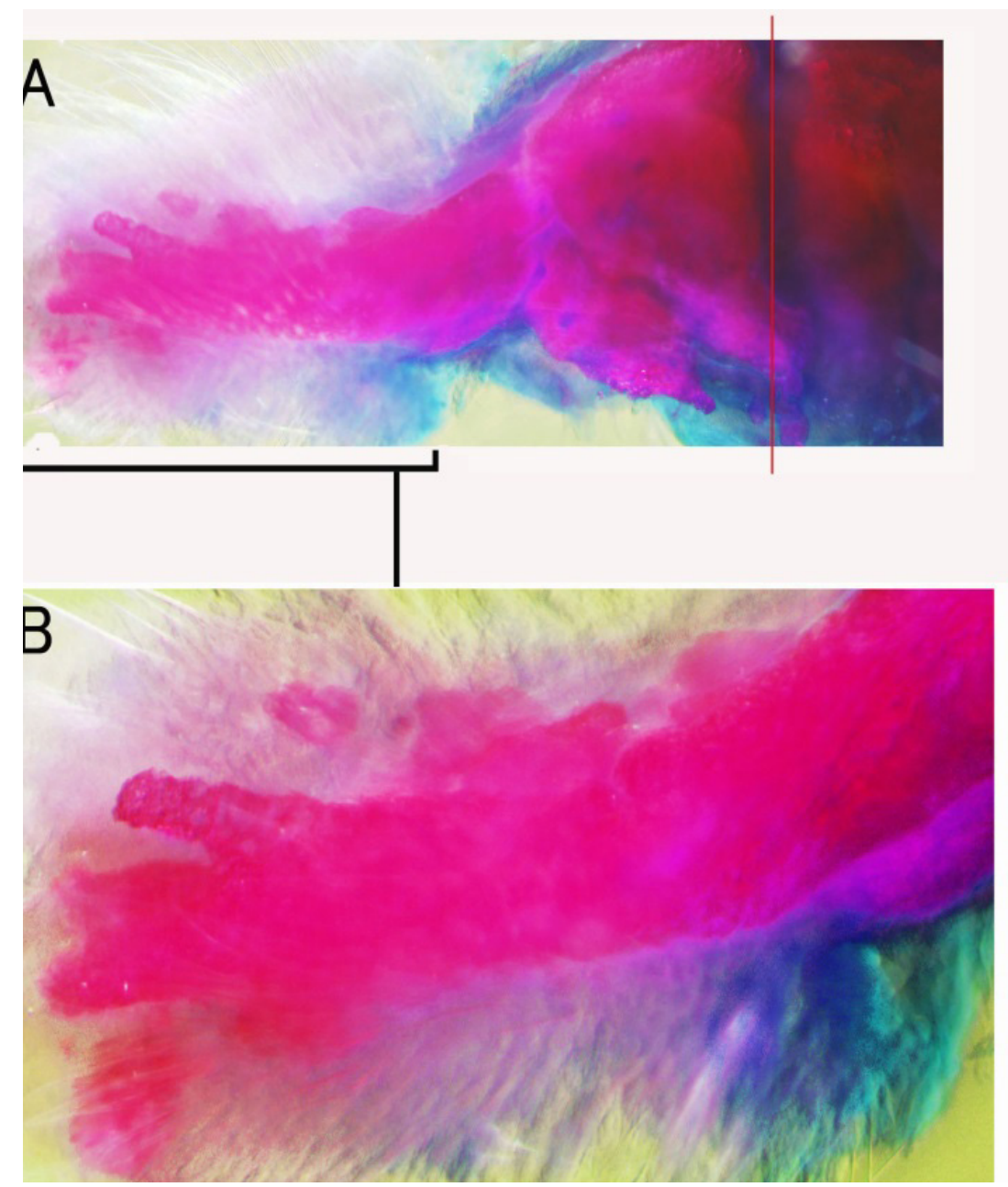

Figure 2. Skeletal pattern formation in a BMP-treated forearm amputated at the palm level showing carpi, metacarpi and digit-like structures. Distal halves of the radius and ulna were also removed at the same time. Alcian blue and alizarin red staining. The red line in A shows the amputation level of the radius and ulna. Long bones were formed on the distal sides of the newly formed radius and ulna-like structures. Enlarged view of the distal region in $\mathrm{A}$ is shown in $\mathrm{B}$.

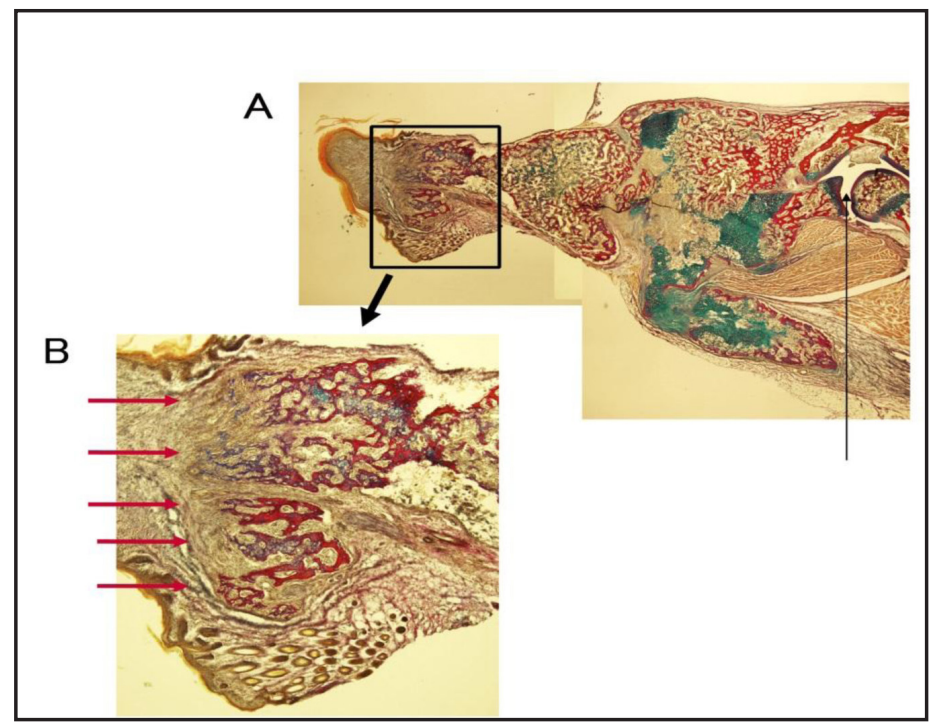

Figure 3. Section of branched long bones stained with Elastica van Gieson and Alcian blue. The thin line in A shows the elbow. The area surrounded by a rectangle is enlarged in B. The red arrows show branched bones. 


\section{Discussion}

Schematics of the experiments are shown in figure 5. In experiment A, distal halves of forearm bones, radius and ulna, were removed and BMP was added in the space. New distal halves of forearm bones were formed. In experiment $B$, hand bones, carpi and metacarpi, were formed on the distal region of the radius-ulna. It is possible that the new bones were formed from dedifferentiated tissues of the forearms. However, they may have been formed from soft tissues of the hand since only bone tissues of the hand were removed. BMP and $\mathrm{Hh}-\mathrm{Ag}$ may induce differentiation of hand soft tissues that remained after hand bone removal to hand bones.

Thus, in experiment $\mathrm{C}$, all hand tissues were removed and gelatin gel containing BMP and $\mathrm{Hh}-\mathrm{Ag}$ was inserted in the epidermis, radius and ulna of the forearm stump. Some small bones other than the newly formed radius and ulna were formed from the forearm tissues, suggesting that these bones were formed from forearm tissues. However, no long thin bones shown in experiment B were observed.
These results suggest that the soft connective tissues and/or muscle tissues of the mouse hand have the potency to form hand bones in the presence of BMP, but these tissues of the forearm have low potency to form hand bones even in the presence of BMP. That is, the acquisition of distal positional values, which is necessary for limb regeneration, may be difficult in the present mouse limb systems.

In mouse digit tips, bone formation is known to be regulated by the intercellular matrix [8] and macrophage systems [9]. These factors seem to promote bone formation also at the forearm level. By activating these factors in the BMP systems, further bone formation in the distal direction and regeneration in mouse limbs will be possible.

\section{Acknowledgment}

I thank Profs. K. Muneoka, T. Endo and A. Sato for their support and advice. We also thank Ms. N. Sagawa for assistance in staining of sections. I also thank the members of Prof. Tamura's laboratory for providing the laboratory.

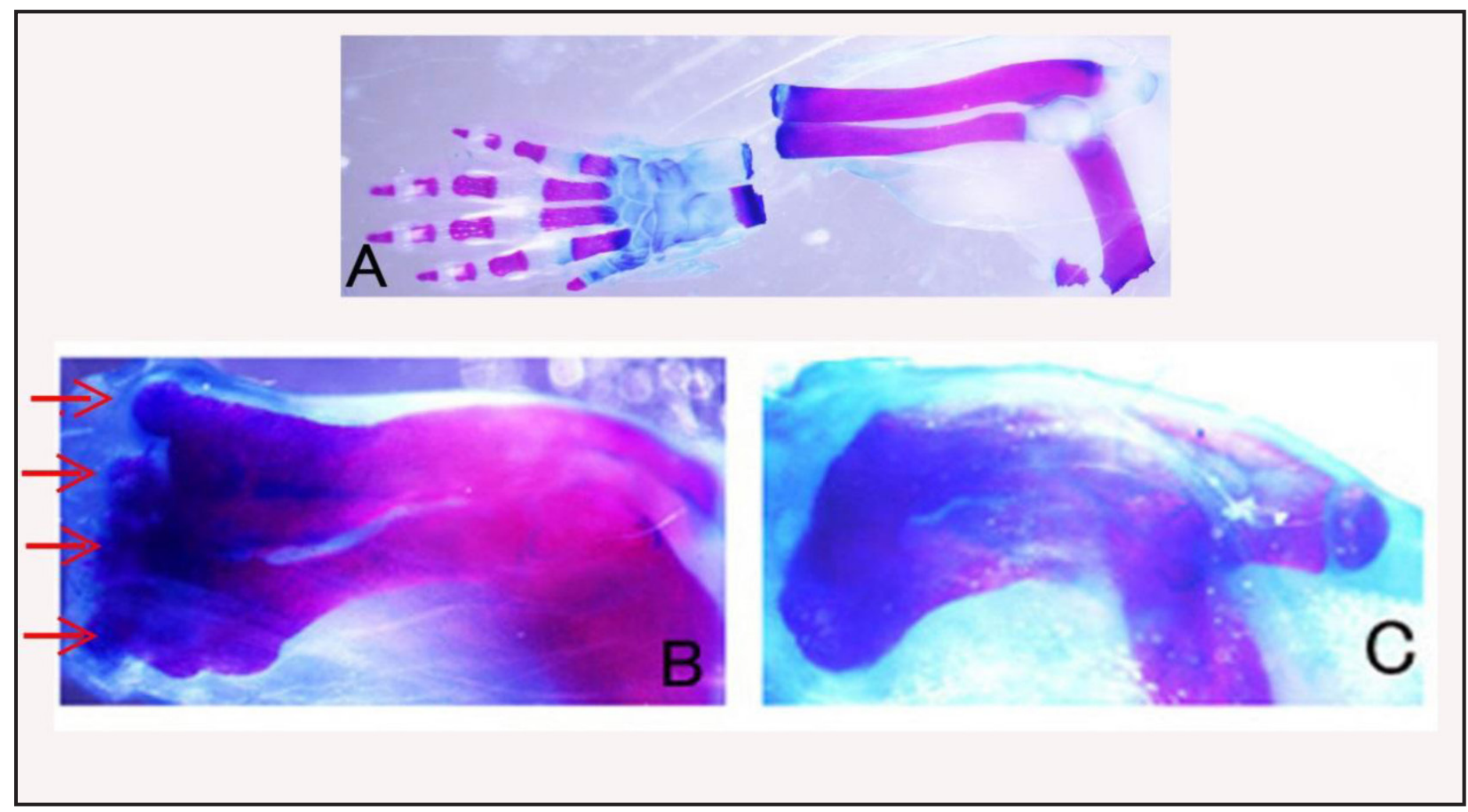

Figure 4A. Skeletal pattern of an arm amputated at the wrist level. Amputated hand and stump forearm were fixed and stained by Alcian blue and Alizarin red to confirm that hand tissues had been removed from the forearm. B: Skeletal pattern of a BMP-treated forearm showing many protrusions (red arrows) at the distal end of the forearm. C: Skeletal pattern of a non-treated forearm. Only hypertrophy (callus) of the amputation site is observed. 


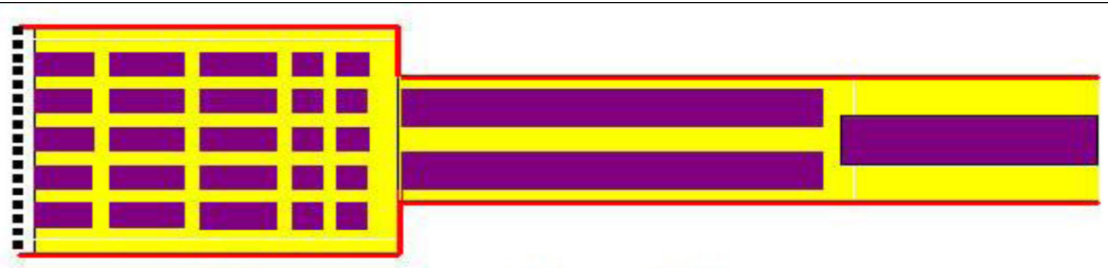

forearm and hand tissues

A

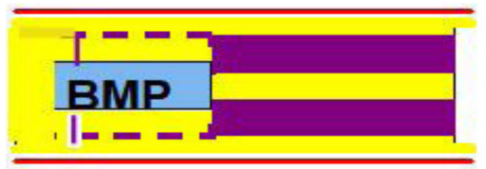

1

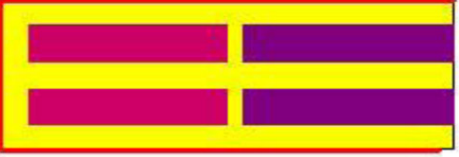

skeletal pattern formation
B

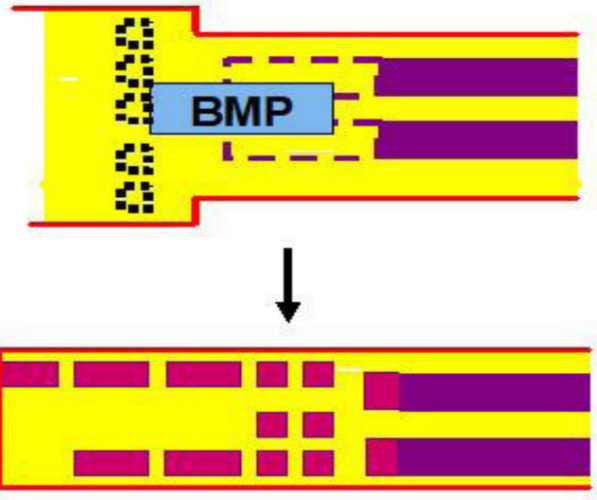

formation of distal skeletal pattern

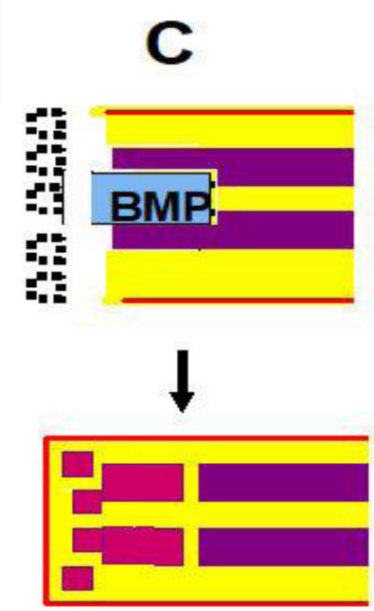

formation of distal skeletal pattern from forearm tissue

Figure 5. Summary of experiments on BMP-induced skeletal pattern formation. The upper diagram shows forearm and hand tissues. Red lines show the epidermis. The yellow area shows soft connective tissues and muscles. The middle diagrams show amputation levels. Violet and black dotted lines show forearm bones removed and hand bones removed, respectively. Gelatin gels containing BMP-7 and a hedgehog agonist were implanted. The lower diagrams show formation of bone patterns. Red rectangles show newly formed bones. A: Skeletal pattern of the forearm the was formed. B: Skeletal pattern of the hand that was formed. C: Many small bones were formed on the distal region of the forearm without hand tissues.

\section{References}

1. Han M, Yang X, Farrington JE, Muneoka K (2003) Digit regeneration is regulated by Msx1 and BMP4 in fetal mice. Development 130: 5123-5132. [crossref]

2. Yu L, Han M, Yan M, Lee EC, Lee J, et al. (2010) BMP signaling induces digit regeneration in neonatal mice. Development 137: 551-559. [crossref]

3. Masaki H, Ide H (2007) Regeneration potency of mouse limbs. Dev Growth Differ 49: 89-98. [crossref]

4. Ide $\mathrm{H}$ (2012) Bone pattern formation in mouse limbs after amputation at the forearm level. Dev Dyn 241: 435-441. [crossref]

5. Ling Y, Han M, Yan M, Lee J, Muneoka K (2012) BMP2 induces segment-specific skeletal regeneration from digit and limb amputation by establishing a new endochondral ossification center. Dev Biol 372: 263-273. [crossref]

6. Parr BA, McMahon AP (1995) Dorsalizing signal Wnt-7a required for normal polarity of D-V and A-P axes of mouse limb. Nature 374: 350-353. [crossref]

7. Miura S, Hanaoka K, Togashi S (2008) Skeletogenesis in Xenopus tropicalis; Characteristic bone development in an anuran amphibian. Bone 43: 901-909. [crossref]

8. Seifert AW, Muneoka K (2018) The blastema and epimorphic regeneration in mammals. Dev Biol 433: 190-199. [crossref]

9. Simkin J, Sammarco MC, Marrero L, Dawson LA, Yan M, et al. (2017) Macrophages are required to coordinate mouse digit tip regeneration. Development 144: 3907-3916. [crossref] 